\title{
The multiple effects of fecal microbiota transplantation on diarrhea-predominant irritable bowel syndrome (IBS-D) patients with anxiety and depression behaviors
}

Hao Lin ${ }^{1,2,9 \dagger}$, Qingqing Guo ${ }^{4 \dagger}$, Zhiyong Wen ${ }^{5 \dagger}$, Songlin Tan ${ }^{5}$, Jie Chen ${ }^{5}$, Lijian Lin ${ }^{6}$, Pengcheng Chen ${ }^{7}$, Jianquan $\mathrm{He}^{8}$, Jianbo Wen ${ }^{5,9^{*}}$ and Ye Chen ${ }^{3,9^{*}}$ (i)

\begin{abstract}
Background: Anxiety and depression are complications in Irritable bowel syndrome (IBS) patients. In this study, we recruited 18 IBS patients with mild-modest anxiety and depression behaviors, and after the screening, we defined the FMT treatment group $(n=9)$ and the control group $(n=9)$. The IBS symptom severity scale (IBS-SSS), Hamilton Anxiety Rating Scale (HAM-A), Hamilton Depression Rating Scale (HAM-D), Irritable Bowel Syndrome Quality of Life (IBS-QOL) and Bristol stool scale (BSS) were evaluated one week before FMT (baseline), one-week-, one-month-, two-month-, and three-month-following FMT. Meanwhile, we determined the SCFAs in the patient's feces and serum and continued the metagenomic analysis of the microorganisms in the patient's feces.
\end{abstract}

Results: The results showed that the patient's anxiety and depression behavior gradually improved with FMT treatment. Moreover, the illness and quality of life had also been relieved significantly. The content of isovaleric acid and valeric acid was significantly reduced in the FMT group compared to the Col group. Metagenomic analysis showed that FMT treatment decreased the abundance of Faecalibacterium, Eubacterium and Escherichia. From KEGG functional analysis, we confirmed that the top five abundant pathways were "bacterial chemotaxis, "flagellar assembly", "glycine, serine and threonine metabolism", "apoptosis", and "bacterial invasion of epithelial cells".

Conclusions: FMT treatment can effectively alleviate the anxiety and depression behaviors of IBS-D patients and reduce the IBS-SSS score, indicating that FMT can improve patients' symptoms. The high throughput sequencing results show that Bifidobacterium and Escherichia play the most critical role in the formation and recovery of IBS-D patients. The GC/MS data indicated that faeces isovaleric acid and valeric acid might be more suitable as a metabolic indicator of IBS-D remission.

Trial registration ChiCTR, ChiCTR1900024924, Registered 3 August 2019, https://www.chictr.org.cn/showproj.aspx? proj $=41676$.

\footnotetext{
*Correspondence: wenjbpx@126.com; yechen@smu.edu.cn

†Hao Lin, Qingqing Guo and Zhiyong Wen contributed equally to this work

${ }^{9}$ State Key Laboratory of Organ Failure Research, Guangdong Provincial Key Laboratory of Gastroenterology, Nanfang Hospital, Southern Medical University, Guangzhou 510515, China

Full list of author information is available at the end of the article
}

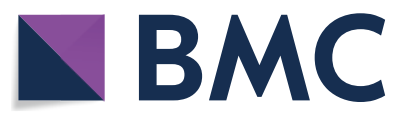

(c) The Author(s) 2021. Open Access This article is licensed under a Creative Commons Attribution 4.0 International License, which permits use, sharing, adaptation, distribution and reproduction in any medium or format, as long as you give appropriate credit to the original author(s) and the source, provide a link to the Creative Commons licence, and indicate if changes were made. The images or other third party material in this article are included in the article's Creative Commons licence, unless indicated otherwise in a credit line to the material. If material is not included in the article's Creative Commons licence and your intended use is not permitted by statutory regulation or exceeds the permitted use, you will need to obtain permission directly from the copyright holder. To view a copy of this licence, visit http://creativecommons.org/licenses/by/4.0/. The Creative Commons Public Domain Dedication waiver (http://creativeco mmons.org/publicdomain/zero/1.0/) applies to the data made available in this article, unless otherwise stated in a credit line to the data. 
Keywords: Irritable bowel syndrome, Fecal microbiota transplantation, Anxiety and depression, Short-chain fatty acids, Metagenome

\section{Background}

In the past decades, the incidence and prevalence of Irritable bowel syndrome (IBS) have increased worldwide [1]. IBS results from a complex interplay between genetic, immunologic, microbial, and environmental factors, making the development of a simple and effective treatment a challenging task. According to the IBS patients' predominant bowel habit, Rome IV categories were build-up for the classification of patients with IBS: diarrhea-predominant (IBS-D), constipation-predominant (IBS-C), mixed diarrhea/constipation (IBS-M), and unclassified (IBS-U). IBS-D may pose a greater diagnostic challenge than the other bowel habit types because patients with chronic or recurrent diarrhea need to consider celiac disease and inflammatory bowel disease [2]. With the deepening of research work on IBS, the progress of the brain-gut axis proved that gut microbes were closely related to emotions [3-5]. Anxiety/depression and IBS are highly prevalent and burdensome conditions, of which co-occurrence is estimated between 44 and $84 \%$ [6]. Liu et al. found that the fecal microbiota profiles in patients with depression were similar to that of IBS-D patients [7]. One hypothesis to explain its mechanism is that the mucosal immune barrier dysfunction induced by microbiota dysfunction leads to increased intestinal permeability, which may be an early step leading to the common pathogenesis of depression and IBS $[8,9]$.

Meanwhile, the primary metabolites of intestinal bacteria also play an essential role in the brain-gut axis. As we know, short-chain fatty acids (SCFAs), the main metabolites produced in the colon by bacterial fermentation of dietary fibers and resistant starch [10], are speculated to play a vital role in neuro-immunoendocrine regulation. Recent findings exhibited that SCFAs have important immunomodulatory functions. Substrate transporters like MCT1 and SMCT1 facilitate the absorption of SCFAs to promote cellular metabolism. Moreover, SCFAs may signal through cell surface G-protein coupled receptors (GPCRs), like GPR41, GPR43, and GPR109A, to activate signaling cascades that control immune functions [11]. Also, the SCFAs can affect cytokine production and migration, cytolytic activity, and epigenetic modulation [12].

As we know, the traditional method of treating IBS aims to reduce symptoms. However, there are many disadvantages and unclear effects in traditional treatment. The emergence of Fecal microbiota transplantation (FMT) treatment, focusing on the microbiome, becoming a fascinating area of research. Nowadays, the understanding of the effectiveness of FMT for IBS, especially the diarrhea-predominant type, is still in its infancy. Due to the differences in various experimental subjects, experimental conditions, and donor conditions, many medical experiments with conflicting results have been caused. Salhy et al. summarized why FMT treatment has different effects on improving the IBS symptoms in different queues, and they believe that FMT is an effective treatment for patients with IBS [13]. Nevertheless, this still needs more work to reveal its mechanism and effectiveness.

In this present study, we recruited IBS-D patients with mild-modest anxiety and depression behaviors, and after the screening, we defined the FMT treatment group (FMT) and the control group (Col). Follow-up was performed to determine the effectiveness of FMT and the improvement of patients' emotion at one week before FMT, one-week-, one-month-, two-month-, and threemonth-following FMT. Meanwhile, we determined the SCFAs in the patient's feces and serum and continued the metagenomic analysis of the microorganisms in the patient's feces. This research is expected to provide evidence for the effectiveness of FMT treatment and reveal the mechanism between FMT and emotions.

\section{Results}

Patient characteristics

The clinical characteristics of patients were shown in Table 1 . The average ages of the Col group and $\mathrm{T}$ group were 50.44 and 44.33 ( $>>0.05$ ), respectively. At baseline, all patients have mild-modest anxiety and depression. Most of the Bristol stool forms of all patients were type 6 $(\mathrm{n}=10,5$ in Col, 5 in T) or type $7(\mathrm{n}=6,4$ in Col, 2 in T). The IBS-SSS ranged from 220 to 320 (avg 287.78), and the IBS-QOL ranged from 32 to 56 (avg 43.72) at baseline.

\section{FMT treatment could relieve patient anxiety and depression}

In this study, FMT treatment significantly alleviated patients' anxiety and depression, which was mainly reflected in the continuous decrease of HAM-A and HAM-D with the increase of treatment time (Fig. 1a and $\mathrm{b})$. The two groups showed significant differences in the first week after FMT treatment in HAM-A evaluation. When the FMT treatment time was prolonged to 1 month, the mean value difference between the two groups was remarkable in both HAM-A and HAM-D 
Table 1 Patient characteristics at baseline

\begin{tabular}{|c|c|c|c|c|c|c|c|c|}
\hline Groups & Patients ID & Age & Gender & HAM-A & HAM-D & IBS-SSS & BSS & IBS-QOL \\
\hline \multirow[t]{9}{*}{$\mathrm{Col}$} & 1 & 69 & Male & 20.5 & 20 & 280 & 6 & 53 \\
\hline & 2 & 59 & Femal & 20.5 & 21.5 & 220 & 6 & 40 \\
\hline & 3 & 65 & Male & 17 & 20.5 & 320 & 6 & 40 \\
\hline & 4 & 48 & Male & 14.5 & 20.5 & 320 & 7 & 42 \\
\hline & 5 & 38 & Male & 23.5 & 22.5 & 300 & 6 & 56 \\
\hline & 6 & 43 & Femal & 28.5 & 25.5 & 340 & 7 & 50 \\
\hline & 7 & 50 & Male & 14.5 & 20.5 & 220 & 6 & 32 \\
\hline & 8 & 42 & Femal & 18 & 25 & 260 & 7 & 48 \\
\hline & 9 & 40 & Femal & 16.5 & 23.5 & 300 & 7 & 36 \\
\hline Avg. & - & $50.44 \pm 10.68$ & - & $19.28 \pm 4.30$ & $22.17 \pm 1.96$ & $284.44 \pm 40.86$ & - & $44.11 \pm 7.61$ \\
\hline \multirow[t]{9}{*}{ FMT } & 10 & 42 & Femal & 24.5 & 24 & 260 & 6 & 51 \\
\hline & 11 & 35 & Male & 15 & 21.5 & 260 & 7 & 48 \\
\hline & 12 & 39 & Male & 23 & 21.5 & 360 & 5 & 48 \\
\hline & 13 & 63 & Male & 16.5 & 27.5 & 260 & 6 & 28 \\
\hline & 14 & 47 & Male & 18.5 & 22 & 300 & 5 & 44 \\
\hline & 15 & 39 & Femal & 17.5 & 22 & 320 & 6 & 40 \\
\hline & 16 & 36 & Femal & 18 & 20.5 & 340 & 7 & 54 \\
\hline & 17 & 58 & Male & 15.5 & 20 & 220 & 6 & 38 \\
\hline & 18 & 40 & Femal & 16.5 & 22 & 300 & 6 & 39 \\
\hline Avg. & - & $44.33 \pm 9.31$ & - & $18.33 \pm 3.10$ & $22.33 \pm 2.11$ & $291.11 \pm 42.28$ & - & $43.33 \pm 7.53$ \\
\hline
\end{tabular}

evaluation, which was maintained in the second and third months. In addition, in the follow-up process, we could observe the mood improvement of the FMTtreated patients. The evaluation of IBS-Qol showed that FMT could improve patients' quality of life at the 1st, 2nd and 3rd month post-FMT-treatment (Fig. 1c). In summary, FMT treatment could relieve anxiety and depression after short-term treatment, and the longer the time, the better the effect. The improvement in the quality of life could also be observed after the FMT treatment.

\section{Remission of IBS symptoms by FMT}

The IBS-SSS score of patients receiving fecal donor material decreased after FMT compared to baseline. The IBS-SSS score of patients in the FMT group was statistically lower than that of patients in the Col group at each time point after treatment. No significant difference in the IBS-SSS score was observed within the Col group between the different time points (Fig. 2a). In addition, FMT could also significantly improve fecal type (Fig. 2b). The stool types of most patients at baseline were between type 5 and type 7 . However, after FMT treatment, a considerable number of patients had fecal type 3 or 4 . In particular, there was no type 7 in the FMT group in the second and third months after completion of therapy.

\section{The FMT treatment caused the changes of SCFAs in feces} and serum

We collected the patients' feces for SCFAs assay. There was no differential SCFAs found between the two groups at baseline. Nevertheless, as illustrated in Fig. 3a, the isovaleric acid and valeric acid levels were sharply reduced in the FMT group compared to the Col group at one-month post-FMT treatment. After KEGG enrichment analysis, we confirmed that isovaleric acid participates in the "Protein digestion and absorption" pathway (ko04974). Moreover, no significant difference in the concentration of SCFAs in serum was found between the two groups at baseline. However, the serum isovaleric acid level was significantly increased in the FMT group (Fig. 3b).

\section{The taxa of the fecal microbiota}

We found that Bacteroidetes and Firmicutes were the main phyla in both groups (Fig. 4a). At the genus level, the abundance of dominant genera, such as Bacteroides and Phocaeicola, were significantly increased by FMT treatment (Fig. 4b). Besides, the level of Bifidobacterium was also showed a significant increasing trend in the FMT group. Conversely, FMT treatment decreased the abundance of Faecalibacterium, Eubacterium and Escherichia (Fig. 4b). 

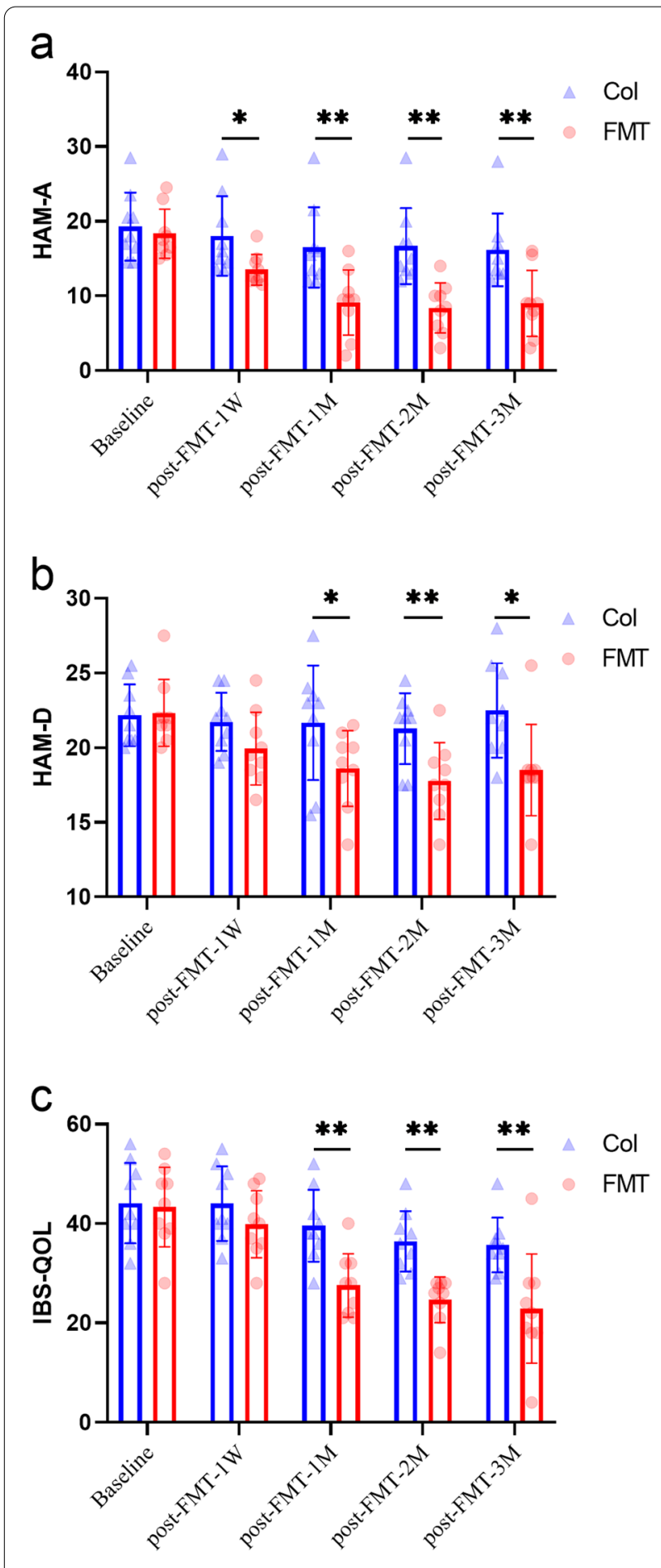

Top abundant pathways from KEGG functional analysis We used the PCA to explore and visualize the difference between the Col and FMT groups. The PCA result showed that samples in both groups were evidently separated (Fig. 5a). Similarly, the apparent differential distributions of KEGG pathways between the two groups
Fig. 1 Effects of FMT on Hamilton Anxiety Rating Scale, Hamilton Depression Rating Scale and Irritable Bowel Syndrome Quality of Life in IBS-D patients with anxiety and depression. a-c shows the HAM-A, HAM-D and IBS-Qol indexes, respectively, of IBS-D patients in Col and FMT. The blue triangles represent the individuals of Col and the red dots represent the individuals of FMT. Bars indicate mean \pm SEM. IBS-D, diarrhea-predominant irritable bowel syndrome; Col, Control group; FMT, FMT treatment group; HAM-A, Hamilton Anxiety Rating Scale; HAM-D, Hamilton Depression Rating Scale; IBS-QOL, Irritable Bowel Syndrome Quality of Life. The abscissa represents the sample collection time. All these evaluation indexes mentioned above were measured at one week prior to FMT (pre-FMT- 1 W), one week following FMT (post-FMT-1 W), one month following FMT (post-FMT-1 M), two months following FMT (post-FMT-2 M), and three months following FMT (post-FMT-3 M). ${ }^{*}, p<0.05$; **, $p<0.01$

were observed (Fig. 5b). From KEGG functional analysis, we found that the top five abundant pathways were "bacterial chemotaxis, "flagellar assembly", "glycine, serine and threonine metabolism", "apoptosis", and "bacterial invasion of epithelial cells". We further screened out the top 40 most abundant genes, which were statistically different in the two groups (Fig. 5c), such as some $A B C$ transporter, malt, $\mathrm{mhpE}$ and $\mathrm{acm}$. In addition, we classified these genes according to the species and functions, and the result showed that most of these genes belonged to Escherichia coli. These genes mainly participate in the process of flagellin, collagen-binding protein, ferric enterobactin receptor and cellulose synthase (Additional file 1: Table S1).

\section{Resistance gene analysis based on the CARD database}

According to the result of resistance gene analysis, we classified all resistance genes. The result showed that the genes belonging to ARO:3,004,480 accounted for the largest proportion (Fig. 6a, Additional file 2: Table S2). The annotated taxonomy of ARO:3,004,480 was Bifidobacterium adolescentis. There were 12 genes classified to ARO:3,000,216 (Escherichia coli), ranking second in all classifications. The resistance gene analysis result identified the top ten genes (or bacteria) with significant differences between the two groups (Fig. 6b). In particular, the abundance of the feature gene of Escherichia was decreased significantly in the FMT group. However, the abundance of the feature gene of Bifidobacterium was significantly increased by FMT treatment.

\section{Discussion}

Our results show that the FMT treatment could improve the IBS-D patients' IBS-SSS scores and quality of life. Meanwhile, it could significantly reduce the scores of the HAM-A and HAM-D of those patients, which might 


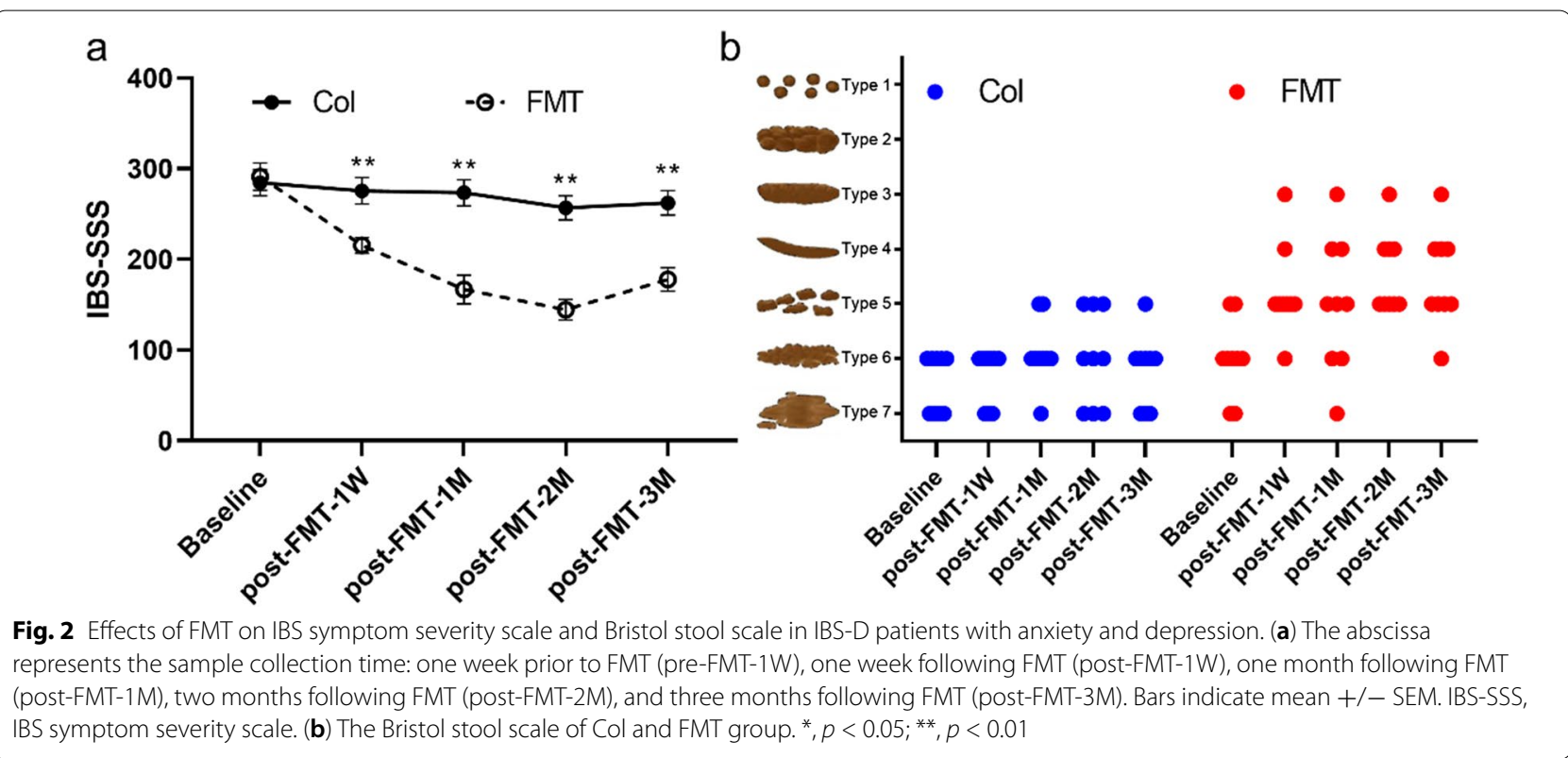

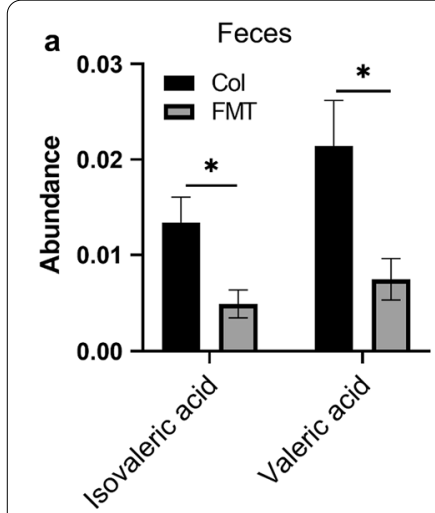

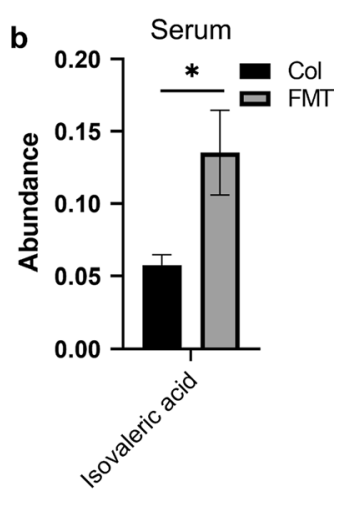

Fig. 3 Effects of FMT on short-chain fatty acids (SCFAs) (a) and (b) shows the level of differential SCFAs in feces and serum, respectively. ${ }^{*}, p<0.05 ;{ }^{* *}, p<0.01$

maintain for some time. Studies on the brain-gut axis also showed that emotions are closely related to the intestinal flora and the environment of the intestine [14]. Intestinal microecological imbalance has been found to be closely related to IBS, inflammatory bowel disease, asthma, emotion (such as anxiety and depression) and other pathological conditions [15]. The results of this study show that FMT treatment could improve patients' IBS-SSS scores and the quality of life. Meanwhile, FMT treatment greatly reduced the patient's anxiety and depression behaviors after a short-term treatment and maintained a long time. There have been many precedents that have proved that FMT could improve the anxiety and depression-like behaviors of experimental

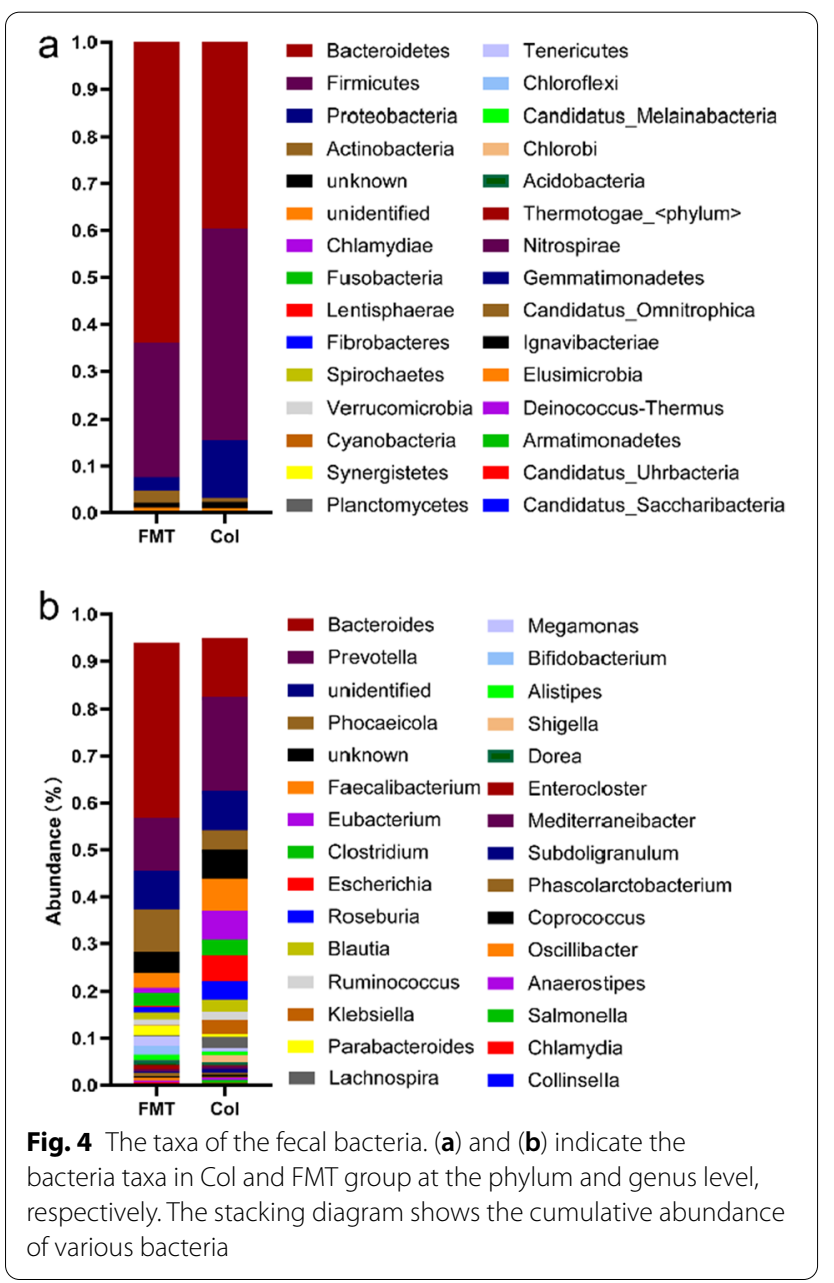



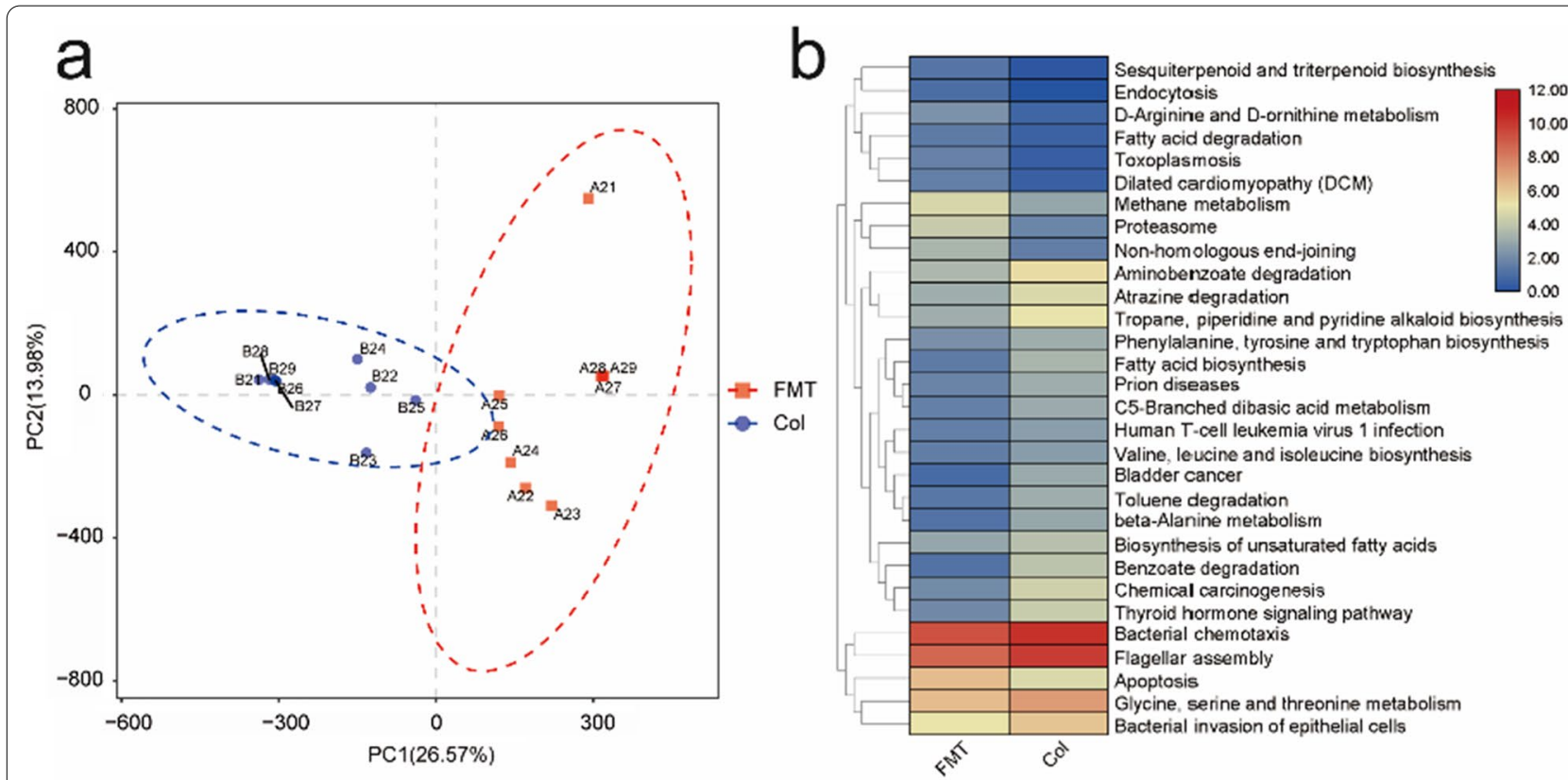

C

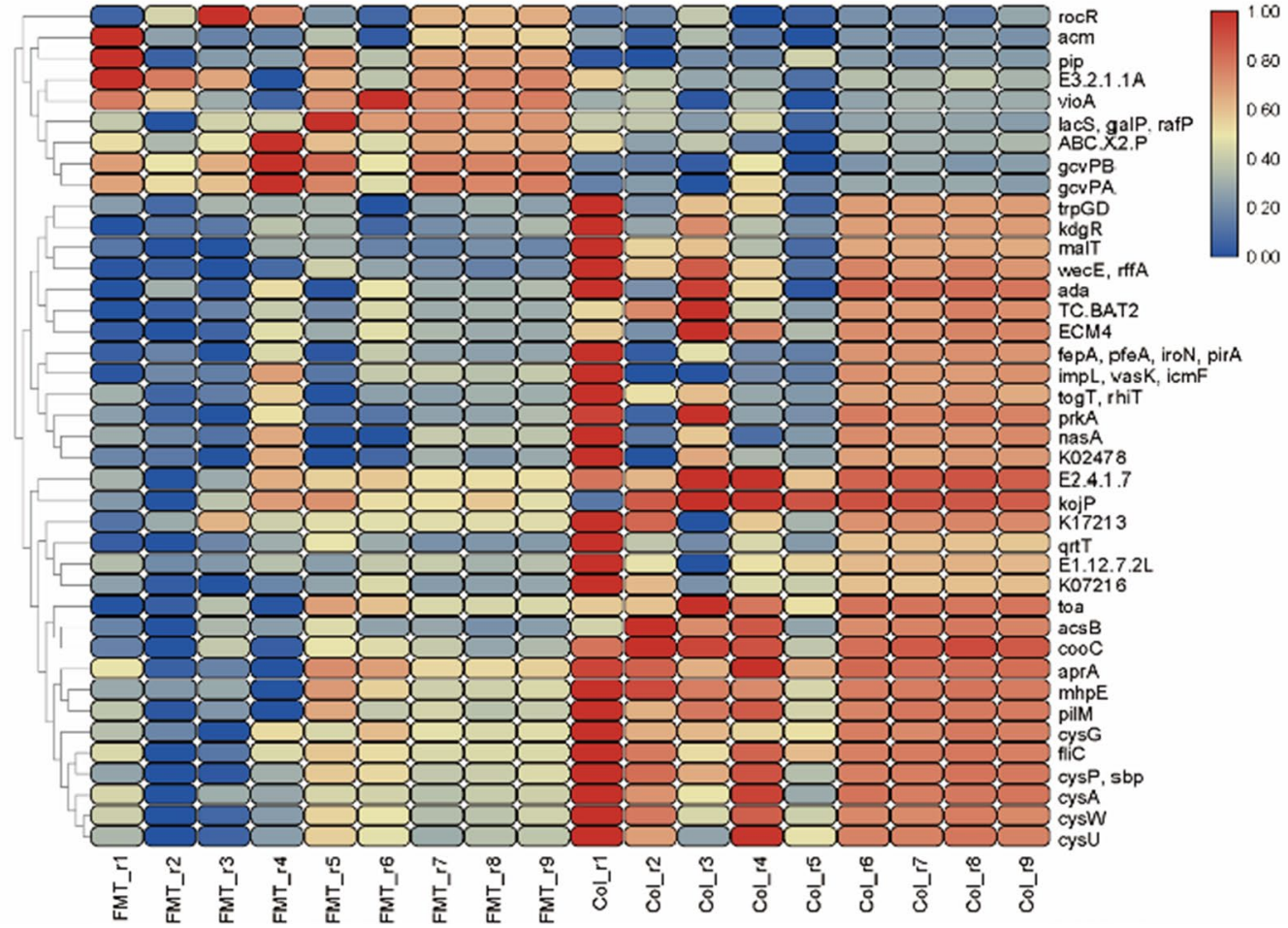

Fig. 5 Results of KEGG functional analysis (a) PCA of the functional genes of the 18 IBS-D patients (PC1, principal component 1; PC2, principal component 2). Red boxes and blue dots represent samples in FMT and Col group, respectively. (b) The abscissa is the group name, the ordinate is the gene function, and the color depth represents the gene function abundance. The figure shows the top 30 KEGG pathways. (c) The heatmap shows the functional gene abundance of the top 40 genes. The color depth represents the gene abundance

subjects [16-19]. Another recent probiotic study that targeted patients with depression showed that subjective depression symptoms improved after probiotic administration and the serum high-sensitivity C-reactive proteins significantly decreased [20]. The benefits of the changes brought about by FMT to patients were obvious. 

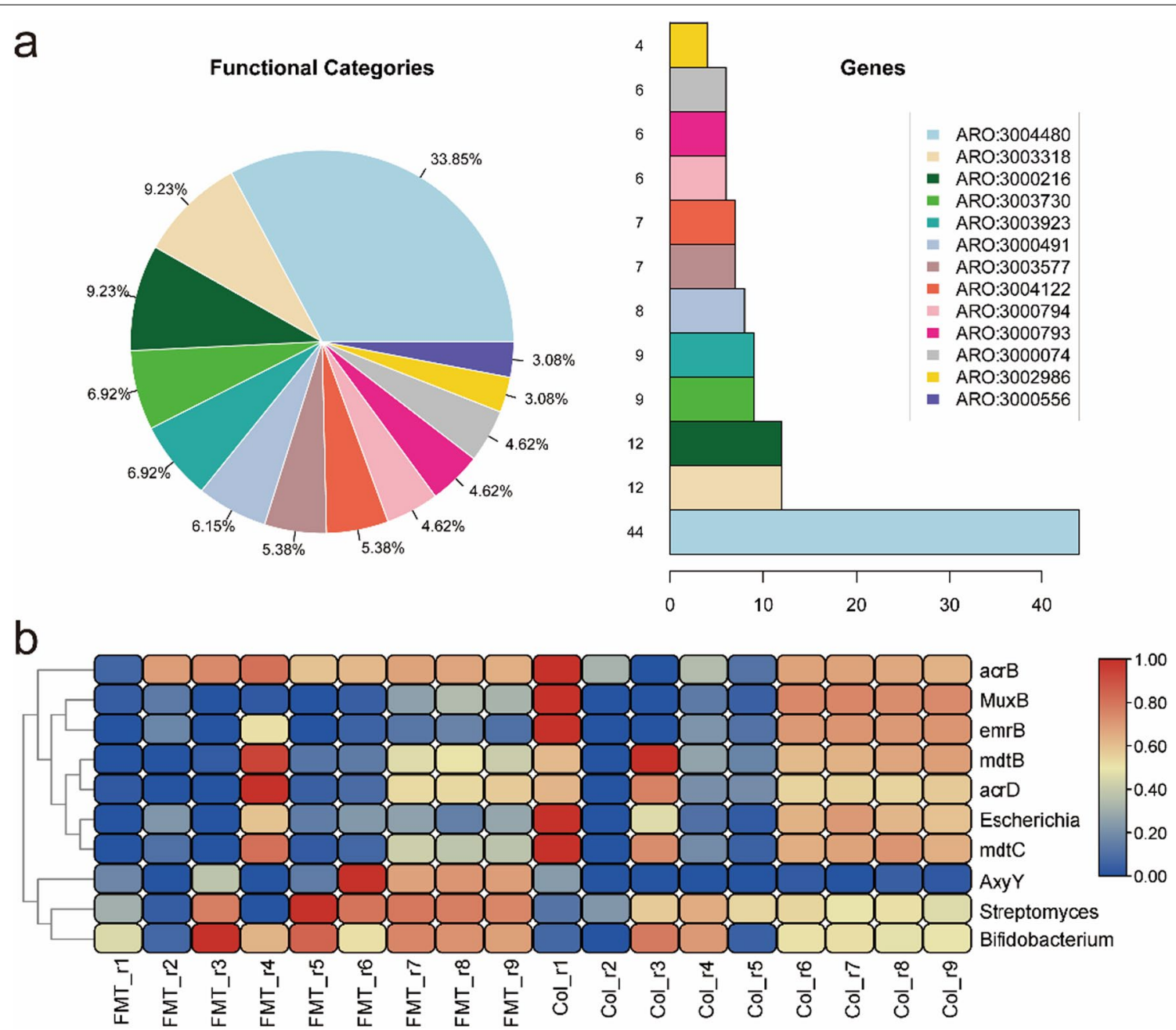

Fig. 6 Resistance gene analysis base on the CARD database (a) The functional categories and genes enriched in CARD database. (b) The top ten genes (or bacteria) with significant differences between the two groups. The heatmap shows the functional gene abundance and the color depth represents the gene abundance

Zhou et al. reported that transplantation of fecal microbiota, from normal mice to antibiotic-pretreated PD mice increased dopamine levels in the recipient PD mice, suggesting that gut microbiota contributed to the neuroprotection for PD [21]. There are evidences that dopamine plays an important role in anxiety and depression [22, 23]. Based on this, we speculate that FMT treatment could contributed to the neuroprotection and alleviate the anxiety- and depression-like behaviors of patients by regulating the dopamine levels. In addition, gut microbes affect the central nervous system by interacting with the vagus nerve [24]. As we know, the vagus nerve is composed of $80 \%$ afferent nerve fibers and $20 \%$ efferent nerve fibers. The afferent nerve fibers of the vagus nerve can be affected by the metabolites of the microbiota, and then bring these gut microbial signals back to the central nervous system [25]. As reported by Breit, the vagus nerve could be affected by long and short chain fatty acids both directly and indirectly, through cellular production of neurotransmitters [26]. Changes in enteric neuron activity perceived by the vagus nerve are essential for mediating satiety, stress, and mood [27, 28]. SCFAs, including acetic acid, propionic acid, butyric acid, isobutyric acid, valeric acid, and isovaleric acid, are end products from intestinal microbial fermentation. Previous studies reported that Bifidobacterium was positively correlated with isovaleric acid, and Escherichia was negatively correlated with isovaleric acid and valeric acid [29, 30]. In this present study, the fecal isovaleric acid and valeric acid were significantly reduced after FMT treatment (Fig. 3), which was correlated with the changes in intestinal flora abundance, such as the decline of Escherichia and the increase of Bifidobacteria (Fig. 4). As we know, valeric acid is commonly found in human feces, it is normal that 
it cannot be detected in the serum [31]. As the regulator, SCFAs might indirectly affect the anxiety and depression through sympathetic nerve. Also, the significant reduction of isovaleric acid and valeric acid in feces might be an obvious sign of the improvement of FMT treatment.

As we know, mucosa is one of the most important parts of the wall of intestine. The mucosa is composed of a single layer of epithelial cells and contains a large number of immune cells. The presence of the epithelial layer is essential for the normal function of the gastrointestinal tract. Meanwhile, the epithelium could effectively resist harmful macromolecules and microorganisms, which act as an important barrier to maintain intestinal health [32]. Impairment of the barrier function could increase permeability, which leads to the abnormal immune response to microorganisms, and luminal antigens had been proposed as an initiating factor in the pathogenesis of chronic human IBS [33, 34]. Previously research reported that one common feature of inflammationassociated microbiotas is increased levels of flagellin, which can occur owing to changes in species composition and/or microbial gene expression [35-39]. The link between elevated microbiota flagellin levels and intestinal inflammation is thought to involve flagellin's ability to activate pro-inflammatory gene expression [40]. In the present study, many genes with significant abundance difference were enriched in "flagellar assembly" pathway, which might reflect enriched levels of motile bacteria that have high ability to penetrate the mucus layer that serves to protect the host against microbial onslaught [40]. Besides, we found that many genes with significant abundance difference were enriched in "bacterial chemotaxis, "glycine, serine and threonine metabolism", "apoptosis" and "bacterial invasion of epithelial cells" pathways. These pathways were closely related to the interaction between epithelial cells and intestinal microorganisms, suggesting that FMT treatment had a positive effect on the reconstruction of intestinal mucosal barrier. Especially, compared with the Col group, FMT treatment significantly reduced the activity of "bacterial invasion of epithelial cells" pathway, indicating that FMT reduced the interaction between pathogenic bacteria (e.g., Shigella, Salmonella, Listeria) and epithelial cells, that is, it reduced the infection risk to a certain extent. According to the genes' annotation, we found that genes related to carbohydrate metabolism (malt), catalytic activity $(m h p E)$, amino acid utilization $(\mathrm{acm})$ and transporters (ABC transporter) were the main functional genes affected by FMT treatment. In addition, most of these genes were found belong to Escherichia coli, which corresponded well with the results of bacterial taxa results. Based on this, we inferred that the changes in these genes are caused by FMT reducing the abundance of
Escherichia coli and increasing Bifidobacterium. And the interaction between bacteria and intestinal epithelial cells might be the key to the pathogenesis of IBS-D.

\section{Conclusions}

The FMT treatment could effectively reduce the IBSSSS score of IBS-D patients and alleviate the anxiety and depression behaviors of these participants, indicating that the FMT therapy might improve the symptoms of IBS-D patients. The GC/MS data indicated that the levels of the isovaleric acid and valeric acid in feces might be suitable metabolic indicators of IBS-D remission. Additionally, the high throughput sequencing results showed that Bifidobacterium and Escherichia may play an important role in the formation and recovery of IBS-D patients. Genes like $A B C$ transporter, malt, $m h p E$, acm, etc. played a key role in pathways like "bacterial chemotaxis, "flagellar assembly", "glycine, serine and threonine metabolism", "apoptosis" and "bacterial invasion of epithelial cells". These pathways may be the main pathways for the interaction between intestinal bacteria and the host.

\section{Materials and methods \\ Participants}

To investigate the gut microbiota in IBS-D patients, we recruited a total of 463 patients with diarrhea-predominant IBS, who also had symptoms of anxiety and depression, from the Pingxiang People's Hospital from Aug. 2019 to Mar. 2021. The enrollment criteria were as follows: ages 20 years or older; IBS-D assessed and diagnosed with the Rome III Diagnostic Criteria (Longstreth et al., 2006); Hamilton Anxiety Rating Scale (HAM-A) score ranges 14-24; Hamilton Depression Rating Scale (HAM-D) score ranges 20-34.

Exclusion criteria were as follows: had abdominal surgery; suffering from human immunodeficiency virus infection; suffering from kidney disease; abnormal liver function indexes; suffering from abnormal thyroid function; history of mental illness; suffering from active infection (excluding Clostridium difficile infection); pregnant woman; people who have taken probiotics, prebiotics, antibiotics orally in the past 2 weeks; cases who are participating in or participated in other clinical trials within 3 months.

Finally, 18 IBS-D patients with symptoms of anxiety and depression were included and randomly divided into two groups: the control (Col) and FMT treatment (FMT).

\section{Donor}

The donor used in this study was screened according to the guidelines for FMT donors in 2019 [41]. He was a 
healthy 36-year-old male, no smoking and drinking, not taking any medication regularly, no class A or class B infectious diseases or infection history and had a normal body mass index. He did not take antibiotics within three months, and had no history of gastrointestinal diseases and mental illness.

Preparation of intestinal bacteria: donor feces were collected and diluted with $500 \mathrm{ml}$ of $0.9 \%$ normal saline. Then, the intestinal bacteria were extracted automatically by an intestinal bacteria extractor (Guangzhou chengge Biotechnology Co., Ltd.). The extracted bacterial solution was then centrifuged at $4000 \mathrm{R} / \mathrm{min}$ for $10 \mathrm{~min}$, and the supernatant was discarded to retain the sediment. After the precipitate is fully stirred, it is divided into capsules in equal quantities, and stored in the refrigerator at -80 ${ }^{\circ} \mathrm{C}$.

\section{Experimental design and sample collection}

All the subjects were randomly divided into two groups, 9 in each group. For group FMT, all the nine included patients received FMT treatment (oral administration) from May 2019 to December 2019. The patients took the intestinal flora capsules 3 times in total, once every other day, 30 capsules each time. For group Col, the patients took the same amount of blank capsules. At the same time, patients' psychiatric symptoms were evaluated by Hamilton Anxiety Rating Scale (HAM-A, https://dcf. psychiatry.ufl.edu/files/2011/05/HAMILTON-ANXIE TY.pdf) and Hamilton Depression Rating Scale (HAMD, https://dcf.psychiatry.ufl.edu/files/2011/05/HAMIL TON-DEPRESSION.pdf) method [42] during the FMT process to analyze the impact of FMT on psychiatric symptoms, and to seek the relationship between microbiota composition and psychiatric symptoms. During the same period, statistics and analysis of Bristol stool scale (BSS) [43, 44], IBS symptom severity scale (IBS-SSS), and Irritable Bowel Syndrome Quality of Life (IBS-QOL) $[45,46]$ were performed to evaluate the effectiveness of FMT treatment. Briefly, the questionnaire of IBS-SSS is meant to register complaint levels related to gastrointestinal symptoms in the form of four questions: (a) Do you suffer from abdominal pain? b) Do you currently suffer from abdominal distention? (c) How satisfied are you with your bowel habits? (d) Please indicate on the line (visual analog scale) below how much your IBS is affecting or interfering with your life in general? A total of 500 points can be reached. A score of up to 75 points is considered as control, $75-175$ points as mild IBS, 175-300 as moderate IBS, and more than 300 as severe IBS. For IBS-QOL, the score ranged from 0 to 100 points and a higher score indicated a better QOL. The score contained 34 questions from 8 aspects: dysphoria, interference with activity, body image, health worry, food avoidance, social reaction, sexual concern and relationship. Stool consistency was assessed by the 7-point Bristol stool scale, a higher score indicating a softer stool. Stool frequency was defined as stools per day. All the evaluation indexes mentioned above were measured at one week prior to FMT (pre-FMT-1 W), one week following FMT (postFMT-1 W), one month following FMT (post-FMT-1M), two months following FMT (post-FMT-2M), and three months following FMT (post-FMT-3 M).

Stool and serum samples were collected one week prior to FMT (pre-FMT-1 W) and one month following FMT (post-FMT-1M). Serum samples were used to detect Short Chain Fatty Acids (SCFAs) through GC/MS technology. The stool sample was divided into two parts, one was used to detect SCFAs, and the other part was used for metagenomic analysis.

\section{Sample preparation and GC/MS analysis}

Stool and serum samples were pretreated according to Zhang report [47]. Briefly, adding $10 \mathrm{mM}$ sodium bicarbonate solution to $1 \mathrm{~g}$ samples; then, adding external standard mixture, tert-butyl methyl ether and $\mathrm{HCl}$ solution to the supernatant after centrifugal extraction; finally, centrifuge again to retain the supernatant for $\mathrm{GC} /$ MS analysis. Each sample were prepared and processed three times.

The SCFAs in the samples were analyzed as free acid form using a Supelco Nukol column $30 \mathrm{~m}$ length, $0.25 \mathrm{~mm}$ internal diameter and $0.25 \mu \mathrm{m}$ of film thickness with the temperatures program as follows: Initial temperature of $40^{\circ} \mathrm{C}$ was held for $1 \mathrm{~min}$, then it was increased to $150^{\circ} \mathrm{C}$ at $30^{\circ} \mathrm{C} / \mathrm{min}$, finally grow up to $220^{\circ} \mathrm{C}$ at $20^{\circ} \mathrm{C} /$ min. A $1 \mu \mathrm{L}$ aliquot of extracted sample was injected in splitless mode (splitless time $1 \mathrm{~min}$ ) at $250^{\circ} \mathrm{C}$, while the transfer line temperature was $280^{\circ} \mathrm{C}$. The used carrier gas was helium and its flow rate maintained at $1 \mathrm{~mL} / \mathrm{min}$ for whole run time. The MS acquisition was carried out in single ion monitoring by apply a proper dwell time ( $20 \mathrm{~ms}$ for each ion monitored) to guarantee a detection frequency of 4 cycle/s. The quantitative determination of SCFAs in each sample was carried out by the ratio between the area abundance of the analytes with the area abundance of respective labeled internal standard (isotopic dilution method). The value of this ratio was named peak area ratio (PAR) and it was used as abundance of each analyte in the quantitative evaluation. The detailed steps were listed in Additional file 3: Table S3.

\section{Metagenomics analysis}

The fecal DNA was extracted using the QIAamp Fast DNA stool minikit (Qiagen, Hilden, Germany) according 
to the manufacturer's instructions. The DNA was first fragmented (using covaris M220). Then, the NEBNext Ultra DNA library prep kit was used to construct the sequencing libraries. The prepared libraries were sequenced by an illumina HiSeq4000 platform with $2 \times 150$ paired-end configuration.

The metagenome data analysis pipeline was shown in Additional file 4: Figure S1. Briefly, the sequence data was firstly evaluated by Trimmomatic software, and the sequencing adapters and sequences with low quality scores were trimmed. High quality short reads were then assembled by the MEGAHIT software (v1.0.6) with default parameters [48]. For functional annotation, MetaGeneMarK was employed to predict the ORFs of the assembled contigs [49]. The predicted ORFs were grouped using CD-HIT with the coverage over $90 \%$ and minimum identity $95 \%$, and finally the non-redundant gene set was obtained [50].

We used BLAST to search for the protein sequences (translated from ORFs) of the predicted Genes in KEGG databases (http://www.genome.jp/kegg/), with $e$-value $<1 \mathrm{e}^{-5}$. The genes with KEGG annotation were assigned into KEGG pathways. For taxonomic assignment we used the Diamond software [51]. Then, based on gene abundance, PCA, PCOA, and PLS-DA analysis were performed by our internal scripts. The difference between different groups were calculated by Kruskal Wallis test. For all analysis details, please refer to the report by Song et al. [52].

\section{Statistical analysis}

Statistical analysis was performed using the SPSS 22 statistical software. Means and standard error of means for the different treatments were calculated and differences between means were determined by t-test on HAM-A, HAM-D, IBS-QOL and IBS-SSS scores. In all cases the level of statistical significance was $\mathrm{P}<0.05$.

\begin{abstract}
Abbreviations
HAM-A: Hamilton Anxiety Rating Scale; HAM-D: Hamilton Depression Rating Scale; IBS-QOL: Irritable Bowel Syndrome Quality of Life; IBS: Irritable bowel syndrome; IBS-D: Diarrhea-predominant; IBS-C: Constipation-predominant; IBS-M: Mixed diarrhea/constipation; IBS-U: Unclassified; SCFAs: Short-chain fatty acids; GPCRs: G-protein coupled receptors; FMT: Fecal microbiota transplantation; Col: Conventional treatment group; ChiCTR: Chinese Clinical Trial Registry; BSS: Bristol stool scale; IBS-SSS: IBS symptom severity scale; IBS-QOL: Irritable Bowel Syndrome Quality of Life; SCFAs: Short Chain Fatty Acids; PAR: Peak area ratio.
\end{abstract}

\section{Supplementary Information}

The online version contains supplementary material available at https://doi. org/10.1186/s12934-021-01720-1.

Additional file 1: Table S1. Gene annotation results.
Additional file 2: Table S2. Genes annotation based on the CARD database.

Additional file 3: Table S3. GC/MS detailed experimental steps.

Additional file 1: Figure S1. Metagenome analysis pipeline.

\section{Acknowledgements}

We thank TreatGut Company (Xiamen, China) for the technical support in the production of enteric capsules.

\section{Authors' contributions}

HL, QG, ZW, YC designed the experiments. HL, ST and JC carried out the experiments. LL, QG and JH prepared the plant materials and analyzed the data. $\mathrm{HL}$, QG, ZW and JW prepared the original manuscript. ST, JC, LL and JW revised the structure of the original manuscript. HL, YC and JW revised the content of the manuscript. All authors read and approved the final manuscript.

\section{Funding}

This work was supported by grants from the National Natural Science Foundation of China (Nos. 81770529 and 82070543), General project of key R \& D plan of Jiangxi Provincial Department of science and Technology (No.:s2020zpyfb0148), Youth Scientific Research Project of Fujian Provincial Health Commission (No.: 2019-1-23) and Startup Fund for Scientific Research of Fujian Medical University (No.: 2018qh1145).

The funding body had no role in study design, the collection, analysis, interpretation of data and manuscript writing.

\section{Availability of data and materials}

We have uploaded the data to the SRA and Metabolights. The data deposited in the SRA and Metabolights is publicly available.

\section{Declarations}

Ethics approval and consent to participate

This study has been registered in the Chinese Clinical Trial Registry (ChiCTR) in 2019 with the access number of ChiCTR1900024924. Ethical clearance was granted by the Ethics Committee of Pingxiang People's Hospital with the approve access of 2019R001-F04.

Consent for publication

All patients gave informed consent and written informed consent was obtained.

\section{Competing interests}

The authors declare that they have no competing interests.

\section{Author details}

1Department of Gastroenterology, Fujian Provincial Hospital South Branch, No. 516, South, Jinrong Road, Cangshan District, Fuzhou 350000, Fujian, China. ${ }^{2}$ Shengli Clinical Medical College, Fujian Medical University, No.134, East Street, Gulou District, Fuzhou 350000, Fujian, China. ${ }^{3}$ Department of Gastroenterology, Integrative Microecology Center, Shenzhen Hospital, Southern Medical University, 1333 New Lake Road, Shenzhen 518100, China. ${ }^{4}$ Department of Intensive Medicine, The First Affiliated Hospital of Fujian Medical University, No.20, Chazhong Road, Taijiang District, Fuzhou 350005, Fujian, China. ${ }^{5}$ Department of Gastroenterology, Affiliated Pingxiang Hospital, Southern Medical University, No. 8, Wugong Mountain Avenue, Development Zone, Pingxiang 337055, Jiangxi, China. ${ }^{6}$ Department of Emergency, Fujian Provincial Hospital, Fujian Medical University, No.134, East Street,Gulou District, Fuzhou 350000, Fujian, China. ${ }^{7}$ Department of Health Management, Fujian Provincial Hospital South Branch, No. 516, South Jinrong Road, Cangshan District, Fuzhou 350000, Fujian, China. ${ }^{8}$ School of medicine, Xiamen University, Xiamen, China. ${ }^{9}$ State Key Laboratory of Organ Failure Research, Guangdong Provincial Key Laboratory of Gastroenterology, Nanfang Hospital, Southern Medical University, Guangzhou 510515, China.

Received: 26 October 2021 Accepted: 11 December 2021

Published online: 28 December 2021 


\section{References}

1. Black CJ, Ford AC. Global burden of irritable bowel syndrome: trends, predictions and risk factors. Nature Reviews Gastroenterology \& Hepatology. 2020;17:473-86.

2. Brandt $L J$, Chey WD, Foxx-Orenstein AE, Schiller LR, Schoenfeld PS, Spiegel BM, Talley NJ, Quigley EM. An evidence-based position statement on the management of irritable bowel syndrome. Am J Gastroenterol. 2009;104(Suppl 1):S1-35.

3. Tillisch K, Mayer EA, Gupta A, Gill Z, Brazeilles R, Le Nevé B, van Hylckama Vlieg JET, Guyonnet D, Derrien M, Labus JS. Brain Structure and Response to Emotional Stimuli as Related to Gut Microbial Profiles in Healthy Women. Psychosom Med. 2017;79:905-13.

4. Lerner A, Neidhöfer S, Matthias T. The Gut Microbiome Feelings of the Brain: A Perspective for Non-Microbiologists. Microorganisms. 2017;5:66.

5. Foster JA. Gut feelings: bacteria and the brain. Cerebrum. 2013;2013:9-9.

6. Simpson CA, Mu A, Haslam N, Schwartz OS, Simmons JG. Feeling down? A systematic review of the gut microbiota in anxiety/depression and irritable bowel syndrome. J Affect Disord. 2020:266:429-46.

7. Liu Y, Zhang L, Wang X, Wang Z, Zhang J, Jiang R, Wang X, Wang K, Liu Z, Xia Z, et al. Similar Fecal Microbiota Signatures in Patients With DiarrheaPredominant Irritable Bowel Syndrome and Patients With Depression. Clin Gastroenterol Hepatol. 2016;14:1602-1611.e1605.

8. Pozuelo M, Panda S, Santiago A, Mendez S, Accarino A, Santos J, Guarner F, Azpiroz F, Manichanh C. Reduction of butyrate- and methane-producing microorganisms in patients with Irritable Bowel Syndrome. Sci Rep. 2015;5:12693.

9. Xu D, Gao J, Gillilland M, Wu X, Song I, Kao JY, Owyang C. Rifaximin alters intestinal bacteria and prevents stress-induced gut inflammation and visceral hyperalgesia in rats. Gastroenterology. 2014;146:484-496.e484.

10. Pascale A, Marchesi N, Marelli C, Coppola A, Luzi L, Govoni S, Giustina A, Gazzaruso C. Microbiota and metabolic diseases. Endocrine. 2018:61:357-71.

11. Parada Venegas D, De la Fuente MK, Landskron G, González MJ, Quera R, Dijkstra G, Harmsen HJM, Faber KN, Hermoso MA. Short Chain Fatty Acids (SCFAs)-Mediated Gut Epithelial and Immune Regulation and Its Relevance for Inflammatory Bowel Diseases. Front Immunol. 2019;10:277-277.

12. Russo E, Giudici F, Fiorindi C, Ficari F, Scaringi S, Amedei A. Immunomodulating activity and therapeutic effects of short chain fatty acids and tryptophan post-biotics in inflammatory bowel disease. Front Immunol. 2019;10:2754

13. El-Salhy M, Hatlebakk JG, Gilja OH, Bråthen Kristoffersen A, Hausken T. Efficacy of faecal microbiota transplantation for patients with irritable bowel syndrome in a randomised, double-blind, placebo-controlled study. Gut. 2020;69:859-67.

14. Panduro A, Rivera-Iñiguez I, Sepulveda-Villegas M, Roman S. Genes, emotions and gut microbiota: The next frontier for the gastroenterologist. World J Gastroenterol. 2017;23:3030-42.

15. Zhang C, Yin A, Li H, Wang R, et al. Dietary modulation of gut microbiota contributes to alleviation of both genetic and simple obesity in children. EBioMed 2015; 2:968-984.

16. Zhao W, Hu Y, Li C, Li N, Zhu S, Tan X, Li M, Zhang Y, Xu Z, Ding Z, et al. Transplantation of fecal microbiota from patients with alcoholism induces anxiety/depression behaviors and decreases brain mGluR1/PKC $\varepsilon$ levels in mouse. BioFactors. 2020;46:38-54.

17. Chinna Meyyappan A, Forth E, Wallace CJK, Milev R. Effect of fecal microbiota transplant on symptoms of psychiatric disorders: a systematic review. BMC Psychiatry. 2020;20:299.

18. Li N, Wang Q, Wang Y, Sun A, Lin Y, Jin Y, Li X. Fecal microbiota transplantation from chronic unpredictable mild stress mice donors affects anxiety-like and depression-like behavior in recipient mice via the gut microbiotainflammation-brain axis. Stress. 2019;22:592-602.

19. Xu Z, Liu Z, Dong X, Hu T, Wang L, Li J, Liu X, Sun J. Fecal microbiota transplantation from healthy donors reduced alcohol-induced anxiety and depression in an animal model of chronic alcohol exposure. Chin J Physiol. 2018;61:360-71.

20. Akkasheh G, Kashani-Poor Z, Tajabadi-Ebrahimi M, Jafari P, Akbari H, Taghizadeh M, Memarzadeh MR, Asemi Z, Esmaillzadeh A. Clinical and metabolic response to probiotic administration in patients with major depressive disorder: a randomized, double-blind, placebo-controlled trial. Nutrition. 2016:32:315-20

21. Zhou Z-L, Jia X-B, Sun M-F, Zhu Y-L, Qiao C-M, Zhang B-P, Zhao L-P, Yang Q. Cui $C$, Chen $X$, et al. Neuroprotection of Fasting Mimicking Diet on
MPTP-Induced Parkinson's Disease Mice via Gut Microbiota and Metabolites. Neurotherapeutics. 2019;16:741-60.

22. Zarrindast MR, Khakpai F. The Modulatory Role of Dopamine in Anxiety-like Behavior. Arch Iran Med. 2015;18:591-603.

23. Dunlop BW, Nemeroff CB. The role of dopamine in the pathophysiology of depression. Arch Gen Psychiatry. 2007;64:327-37.

24. Ma Q, Xing C, Long W, Wang HY, Liu Q, Wang R-F. Impact of microbiota on central nervous system and neurological diseases: the gut-brain axis. J Neuroinflam. 2019;16:53.

25. Bonaz B, Bazin T, Pellissier S. The vagus nerve at the interface of the microbiota-gut-brain axis. Front Neurosci. 2018;12:49.

26. Breit S, Kupferberg A, Rogler G, Hasler G. Vagus nerve as modulator of the brain-gut axis in psychiatric and inflammatory disorders. Front Psychiatry. 2018:9:44

27. Forsythe P, Bienenstock J, Kunze WA. Vagal pathways for microbiome-braingut axis communication. Adv Exp Med Biol. 2014;817:115-33.

28. Browning KN, Verheijden S, Boeckxstaens GE. The vagus nerve in appetite regulation, mood, and intestinal inflammation. Gastroenterology. 2017;152:730-44

29. Hemalatha R, Ouwehand AC, Saarinen MT, Prasad UV, Swetha K, Bhaskar V. Effect of probiotic supplementation on total lactobacilli, bifidobacteria and short chain fatty acids in 2-5-year-old children. Microb Ecol Health Dis. 2017:28:1298340-1298340

30. Liao X, Shao Y, Sun G, Yang Y, Zhang L, Guo Y, Luo X, Lu L. The relationship among gut microbiota, short-chain fatty acids, and intestinal morphology of growing and healthy broilers. Poult Sci. 2020;99:5883-95.

31. Høverstad T, Fausa O, Bjørneklett A, Bøhmer T. Short-chain fatty acids in the normal human feces. Scand J Gastroenterol. 1984;19:375-81.

32. Chichlowski M, Hale LP. Bacterial-mucosal interactions in inflammatory bowel disease: an alliance gone bad. Am J Physiol Gastrointest Liver Physiol. 2008:295:G1139-49.

33. Burns G, Carroll G, Mathe A, Horvat J, Foster P, Walker MM, Talley NJ, Keely S. Evidence for Local and Systemic Immune Activation in Functional Dyspepsia and the Irritable Bowel Syndrome: A Systematic Review. Official journal of the American College of Gastroenterology | ACG 2019; 114.

34. Collins SM. A case for an immunological basis for irritable bowel syndrome. Gastroenterology. 2002;122:2078-80

35. Chassaing B, Koren O, Goodrich JK, Poole AC, Srinivasan S, Ley RE, Gewirtz AT. Dietary emulsifiers impact the mouse gut microbiota promoting colitis and metabolic syndrome. Nature. 2015:519:92-6.

36. Chassaing B, Van de Wiele T, De Bodt J, Marzorati M, Gewirtz AT. Dietary emulsifiers directly alter human microbiota composition and gene expression ex vivo potentiating intestinal inflammation. Gut. 2017;66:1414-27.

37. Cullender TC, Chassaing B, Janzon A, Kumar K, Muller CE, Werner JJ, Angenent LT, Bell ME, Hay AG, Peterson DA, et al. Innate and adaptive immunity interact to quench microbiome flagellar motility in the gut. Cell Host Microbe. 2013;14:571-81.

38. Chassaing B, Ley RE, Gewirtz AT. Intestinal epithelial cell toll-like receptor 5 regulates the intestinal microbiota to prevent low-grade inflammation and metabolic syndrome in mice. Gastroenterology. 2014;147:1363-1377.e1317.

39. Chassaing B, Koren O, Carvalho FA, Ley RE, Gewirtz AT. AIEC pathobiont instigates chronic colitis in susceptible hosts by altering microbiota composition Gut 2014:63:1069-80.

40. Tran HQ, Ley RE, Gewirtz AT, Chassaing B. Flagellin-elicited adaptive immunity suppresses flagellated microbiota and vaccinates against chronic inflammatory diseases. Nat Commun. 2019;10:5650.

41. Cammarota G, laniro G, Kelly CR, Mullish BH, Allegretti JR, Kassam Z, Putignani L, Fischer M, Keller JJ, Costello SP, et al. International consensus conference on stool banking for faecal microbiota transplantation in clinical practice. Gut. 2019;68:2111-21.

42. Hamilton M. The assessment of anxiety states by rating. Br J Med Psychol. 1959;32:50-5.

43. Lewis SJ, Heaton KW. Stool form scale as a useful guide to intestinal transit time. Scand J Gastroenterol. 1997:32:920-4.

44. Choi YJ, Park YS, Kim N, Kim YS, Lee SM, Lee DH, Jung HC. Gender differences in ghrelin, nociception genes, psychological factors and quality of life in functional dyspepsia. World J Gastroenterol. 2017;23:8053-61.

45. Mönnikes H. Quality of life in patients with irritable bowel syndrome. J Clin Gastroenterol. 2011;45(Suppl):S98-101.

46. Liang AM, Tien Y. Quality of Life in Irritable Bowel Syndrome: A Narrative Overview. J Arch Mil Med. 2016:4:e36624. 
47. Zhang S, Wang H, Zhu M-J. A sensitive GC/MS detection method for analyzing microbial metabolites short chain fatty acids in fecal and serum samples. Talanta. 2019;196:249-54.

48. Li D, Liu C-M, Luo R, Sadakane K, Lam T-W. MEGAHIT: an ultra-fast singlenode solution for large and complex metagenomics assembly via succinct de Bruijn graph. Bioinformatics. 2015;31:1674-6.

49. Zhu W, Lomsadze A, Borodovsky M. Ab initio gene identification in metagenomic sequences. Nucleic Acids Res. 2010;38:e132.

50. Fu L, Niu B, Zhu Z, Wu S, Li W. CD-HIT: accelerated for clustering the nextgeneration sequencing data. Bioinformatics. 2012;28:3150-2.

51. Bağcl C, Patz S, Huson DH. DIAMOND+MEGAN: fast and easy taxonomic and functional analysis of short and long microbiome sequences. Curr Protocols. 2021;1:e59.

52. Song B, Li Z, Li S, Zhang Z, Fu Q, Wang S, Li L, Qi S. Functional metagenomic and enrichment metatranscriptomic analysis of marine microbial activities within a marine oil spill area. Environ Pollut. 2021;274:116555.

\section{Publisher's Note}

Springer Nature remains neutral with regard to jurisdictional claims in published maps and institutional affiliations.

- fast, convenient online submission

- thorough peer review by experienced researchers in your field

- rapid publication on acceptance

- support for research data, including large and complex data types

- gold Open Access which fosters wider collaboration and increased citations

- maximum visibility for your research: over $100 \mathrm{M}$ website views per year

At BMC, research is always in progress.

Learn more biomedcentral.com/submissions 